\title{
CORRECCIONES DE ENSAYOS QUÍMICOS EN UNA SERIE DE NODOS ENLAZANTES POR MULTIPLICADORES DE LAGRANGE
}

\section{CORRECTIONS OF CHEMICAL TESTS IN A SERIES OF NODES BASONAT BY LAGRANGE MULTIPLIER}

\author{
Ing. Edgar Ademar Pérez Matos ${ }^{1}$, Lic. Andrés Collante Huanto² ${ }^{2}$ Ing. M.Sc. Efraín E. \\ Castillo Alejos ${ }^{3}$ \\ RESUMEN
}

El presente trabajo mostrará una variedad de pasos secuenciales con el fin de obtener la corrección del análisis químico (leyes), en los flujos de una serie de nodos (celdas) enlazantes mediante Función Generalizada; quien se adecua perfectamente al análisis de los procesos de flotación de índole industrial en el procesamiento de minerales. De donde obtenido las leyes (contenido metálico) de los análisis químico ya realizado se procede a su corrección respectiva; quien involucra todo un procedimiento desde su diagrama de flujo, sus leyes; para luego calcular los flujos normalizados, los multiplicadores de Lagrange (optimizador de errores), y las correcciones que conllevan finalmente a lo deseado.

Palabras clave.- Diagrama de flujo, Multiplicadores de lagrange.

\begin{abstract}
The present work will display sequential steps in order to obtain correct of the chemical assays in the flows of a series of nodes (cells). Basonat function through widespread; who is perfectly suited to the analysis of the processes of industrial nature float in mineral processing. Where obtained the assay valves (metal content) of the chemical analysis has already been made are appropriate to their respective correction; who involves a whole procedure since its flow diagram, its laws; for then to calculate the standardized flows, the Lagrange multiplier (optimizer for errors), and the corrections that finally lead to the desired.
\end{abstract}

Keywords.- Flow diagram, Lagrange Multiplier.

\section{INTRODUCCIÓN}

Se sabe que en toda Planta de procesamiento de minerales, el funcionamiento es continuo (Etapa de flotación de minerales), y por consiguiente no se puede mantenerlo constante por mucho tiempo. Entonces al muestrear se insertan involuntariamente errores en los datos, ya sea por el proceso en sí o por manipuleo; luego el objetivo será tratar de corregir los datos para recién proceder a su balance metalúrgico respectivo (que nos reporta el contenido metálico a recuperar). Las correcciones a calcular serán las mínimas posibles, debido a que los errores se les aproximarán a cero. A continuación se mostrará una variedad de pasos secuenciales con el fin de calcular la corrección del análisis químico (leyes) obtenido de los flujos en una serie de nodos enlazantes mediante la función generalizada. Entonces lo que el modelamiento básicamente hará, es tratar de que

\footnotetext{
${ }^{1}$ Universidad Nacional de Ingeniería, ${ }^{2}$ Universidad Nacional de Ingeniería, ${ }^{3}$ Universidad Nacional de
} Ingeniería Obtención de los flujos normalizados 
los errores sean distribuidos a través del sistema, de acuerdo a la función generalizada, que más adelante será deducida, analizada y aplicada a nivel industrial.

Con los flujos y sus respectivos análisis químico (leyes), registrados según la figura 1 y la tabla 1 .

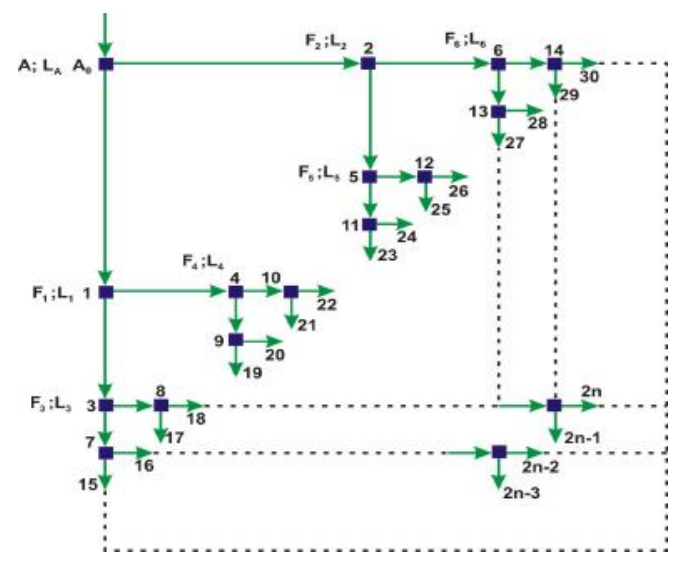

- Flujos ;

A: Representa al primer Flujo Principal (alimento).

$\left\{F_{1}, F_{2}, F_{3}, \ldots, F_{2 n}\right\}:$ Representa a los flujos secundarios.

\section{- Nodos;}

$\mathrm{A}_{0}$ : Representa al primer Nodo Principal.

$\left\{1,2,3, \ldots, \frac{2 n-2}{2}\right\}:$ Representa a los nodos secundarios.

(n-1): Representa al último nodo, también al número de nodos secundarios.

Cada análisis químico en los flujos, arroja los siguientes datos:

Fig. 1 Esquema del circuito.

Tabla 1. Análisis químico a corregir.

\begin{tabular}{|c|c|c|c|c|c|}
\hline+ & & & EYES & & \\
\hline FLUJOS(u) & $\mathrm{i}: 1$ & $\mathrm{i}: 2$ & $\mathrm{i}: 3$ & $\ldots \ldots \ldots \ldots \ldots$ & $\mathrm{i}: \mathrm{M}$ \\
\hline $\mathrm{A}$ & $L_{A}^{(1)}$ & $L_{A}^{(2)}$ & $L_{A}^{(3)}$ & …........... & $L_{A}^{(M)}$ \\
\hline $\mathrm{F}_{1}$ & $L_{1}^{(1)}$ & $L_{1}^{(2)}$ & $L_{1}^{(3)}$ & $\ldots \ldots+\cdots \cdots$ & $L_{1}^{(M)}$ \\
\hline $\mathrm{F}_{2}$ & $L_{2}^{(1)}$ & $L_{2}^{(2)}$ & $L_{2}^{(3)}$ & $\cdots$ & $L_{2}^{(M)}$ \\
\hline $\mathrm{F}_{3}$ & $L_{3}^{(1)}$ & $L_{3}^{(2)}$ & $L_{3}^{(3)}$ & (nown. & $L_{3}^{(M)}$ \\
\hline . & . & . & . & . & . \\
\hline . & . & . & $\cdot$ & - & . \\
\hline · & . & - & . & - & . \\
\hline$F_{2 n}$ & $L_{2 n}^{(1)}$ & $L_{2 n}^{(2)}$ & $L_{2 n}^{(3)}$ & $\ldots \ldots \ldots \ldots$ & $L_{2 n}^{(M)}$ \\
\hline
\end{tabular}

Donde:

$$
\begin{aligned}
& \mathrm{I}=\{1,2,3,4, \ldots, \mathrm{M}\}: \text { Representa las leyes } \\
& \mathrm{u}=\left\{\mathrm{A}, \mathrm{F}_{1}, \mathrm{~F}_{2}, \mathrm{~F}_{3}, \ldots, \mathrm{F}_{2 \mathrm{n}}\right\}: \text { Representa los }
\end{aligned}
$$
flujos.

Además:

$(2 n+1):$ Representa al número de flujos.
Constituir las ecuaciones de Balance de Masa (Ecuaciones de Flujo y Leyes).

Normalizar las ecuaciones anteriores (Dividendo por el flujo "A").

Constituir las ecuaciones de error con los flujos normalizados ( Q). 
Identificar la Función $f\left(\phi_{i}\right)$.

Sintetizando estas últimas ecuaciones e introduciendo sus respectivas leyes (i); además elevando al cuadrado resulta: Función generalizada.

\section{FUNCION GENERALIZADA}

Que involucra flujos normalizados, leyes y sus respectivos errores en cada nodo (celda).

$f(\phi i)=\sum_{i=1}^{M}\left\{\left(\Omega_{A, 2}^{(i)}-\Omega_{A, 2}^{(i)} \cdot \phi_{1}\right)^{2}+\sum_{N=1}^{n-1}\left(\Omega_{N, 2 N+2}^{(i)} \cdot \phi_{N}-\Omega_{2 N+1,2 N+2}^{(i)} \cdot \phi_{2 N+1}\right)^{2}\right\}$

$f\left(\phi_{i}\right)$ : Será la función que represente a la suma de los errores ( Q) elevado al cuadrado.

Derivar parcialmente la función $\mathrm{f}\left(\phi_{\mathrm{i}}\right)$, con respecto a cada flujo normalizado correspondiente e igualándolos a cero; esto propicia hallar flujos normalizados mínimos que hacen que el error tienda a cero.

Deducción de los " $n$ " flujos normalizados de sub índice impar. Se procede a derivar parcialmente la función $f\left(\phi_{i}\right)$, inicio del hallazgo de todos los flujos normalizados.

$$
\frac{\partial f\left(\phi_{i}\right)}{\partial \phi_{1}}=0
$$

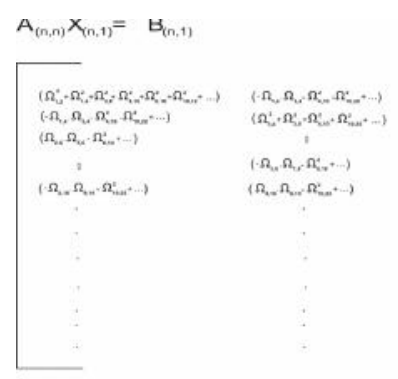

$$
\left\{\begin{array}{l}
+\phi_{1}\left(\Omega_{1,2}^{2}+\Omega_{1,4}^{2}+\Omega_{2,6}^{2}+\Omega_{4,10}^{2}+\Omega_{6,14}^{2}+\Omega_{10,22}^{2}+\ldots\right) \\
+\phi_{3}\left(-\Omega_{1,4} \cdot \Omega_{3,4}-\Omega_{4,10}^{2}-\Omega_{10,22}^{2}-\ldots\right) \\
+\phi_{5}\left(\Omega_{2,6} \cdot \Omega_{5,6}+\Omega_{6,14}^{2}+\ldots\right) \\
+\phi_{9}\left(-\Omega_{4,10} \cdot \Omega_{9,10}-\Omega_{10,22}^{2}-\ldots\right) \\
+\phi_{13}\left(\Omega_{6,14} \cdot \Omega_{13,14}+\ldots\right) \\
+\phi_{21}\left(-\Omega_{10,22} \cdot \Omega_{21,22}+\ldots\right) \\
=-\Omega_{A, 2} \cdot \Omega_{1,2}+\Omega_{2,6}^{2}+\Omega_{6,14}^{2} \cdot \phi_{9}+\ldots
\end{array}\right.
$$

$$
\begin{aligned}
& \frac{\partial f\left(\phi_{i}\right)}{\partial \phi_{3}}=0 \\
& \left\{\begin{array}{l}
+\emptyset_{1}\left(-\Omega_{1,1}, \Omega_{2,4}-\Omega_{\Lambda_{, 10}}^{2}-\Omega_{10,22}^{2}+\ldots\right) \\
+\emptyset_{3}\left(\Omega_{3,4}^{2}+\Omega_{3,9}^{2}+\Omega_{4,10}^{2}+\Omega_{10,22}^{2}+\ldots\right) \\
+\emptyset_{7}\left(-\Omega_{3,9} \Omega_{7,8}-\Omega_{\Omega, 18}^{2}+\ldots\right) \\
+\emptyset_{9}\left(\Omega_{10,22}^{2}+\ldots\right) \\
+\emptyset_{17}\left(-\Omega_{8,18} \Omega_{1,18}+\ldots\right) \\
\mid \emptyset_{21}\left(\Omega_{10,22} \cdot \Omega_{11,22} \| \ldots\right) \\
=\quad-\Omega_{\Omega, 18}^{2}+\ldots
\end{array}\right.
\end{aligned}
$$

De las " $\mathrm{n}$ " derivadas parciales obtenidas, se procede a formar la siguiente Ecuación Lineal Matricial: $A(n, n) X(n, 1)=B(n, 1)$, de quien se obtiene los " $\mathrm{n}$ " flujos normalizados (de sub índice impar) respectivamente, quedando por calcular los "n" flujos (de sub índice par) restantes.

Y que valiéndose de las ecuaciones del paso (1.3) será posible hallarlos.

Ecuación Lineal Matricialmente, de los Flujos Normalizados.

Seguidamente se procede a despejar la ecuación matricial: $\mathrm{X}_{\mathrm{n}, 1}=\mathrm{A}_{\mathrm{n}, \mathrm{n}} \cdot \mathrm{B}_{\mathrm{n}-1}$; dando inicio el hallazgo de los flujos normalizados.

\section{OBTENCION DE LOS MULTIPLICADORES DE LAGRANGE}

Cálculo de los errores: $\mathrm{M}$ 
Definir las Correcciones:

Corrección $=$ Datos - Datos corregidos .

Reemplazando las ecuaciones del paso (2.2) en (2.1) y además considerando que en las leyes ya corregidas el error es nulo. Con lo cual obtenemos los errores en función de las correcciones. $\left(\mathrm{L}_{\mathrm{i}}\right)$

Definir la función Lagrangiana.

$\mathrm{L}(\mathrm{x}, \lambda)=\mathrm{f}(\mathrm{x})-\left[\lambda \cdot \mathrm{g}(\mathrm{x})+\lambda_{1} \cdot \mathrm{g}_{1}(\mathrm{x})+\lambda_{2} \cdot \mathrm{g}_{2}(\mathrm{x})+\ldots+\right.$ $\left.\lambda_{\mathrm{n}-1} \cdot \mathrm{g}_{\mathrm{n}-1}(\mathrm{x})\right]$

Donde:

$\mathrm{L}(\mathrm{x}, \lambda)$ : función lagrangiana $\mathrm{f}(\mathrm{x}): \mathrm{L}_{\mathrm{A}}^{2}+\mathrm{L}_{1}^{2}+\mathrm{L}_{2}^{2}+\ldots \quad \mathrm{L}_{2 \mathrm{n}}{ }^{2}$; función objetivo.

$\lambda_{\mathrm{i}}: \lambda, \lambda_{1}, \lambda_{2} \ldots \lambda_{\mathrm{n}-1} ;$ multiplicadores de Lagrange

$\mathrm{g}_{\mathrm{i}}^{(\mathrm{x})}$ : Función restricta (viene del paso 2.3)

$$
\begin{aligned}
& \mathrm{g}^{(\mathrm{x})}=\mathrm{M}-\mathrm{L}_{\mathrm{A}}+\left(\mathrm{L}_{1} \cdot \emptyset_{1}+\mathrm{L}_{2} \cdot \emptyset_{2}\right) \\
& \mathrm{g}_{1}^{(\mathrm{x})}=\mathrm{M}_{1}-\mathrm{L}_{1} \cdot \emptyset_{1}+\left(\mathrm{L}_{3} \cdot \emptyset_{3}+\mathrm{L}_{4} \cdot \emptyset_{4}\right. \\
& \mathrm{g}_{2}{ }^{(\mathrm{x})}=\mathrm{M}_{2}-\mathrm{L}_{2} \cdot \emptyset_{2}+\left(\mathrm{L}_{5} \cdot \emptyset_{5}+\mathrm{L}_{6} \cdot \emptyset_{6}\right)
\end{aligned}
$$

Derivar parcialmente $\mathrm{L}(\mathrm{x}, \lambda)$ respecto a los multiplicadores de Lagrange y las correcciones e igualarlos a cero.

Estas correcciones harán que la función objetivo tome un valor mínimo sujeto a las ecuaciones restrictas.

Derivando parcialmente respecto a los n" multiplicadores de lagrange.

$\frac{\partial L(\mathrm{x}, \lambda)}{\partial \lambda}=0 ; \quad \mathrm{M}-\mathrm{L}_{\mathrm{A}}+\left(\mathrm{L}_{1} \emptyset_{1}+\mathrm{L}_{2 .} \emptyset_{2}\right)=0$

$\frac{\partial L(\mathrm{x}, \lambda)}{\partial \lambda_{1}}=0 ; \quad \mathrm{M}_{1}-\mathrm{L}_{1} \varnothing_{1}+\left(\mathrm{L}_{3} . \varnothing_{3}+\mathrm{L}_{4} . \varnothing_{4}\right)=$ 0

Derivando parcialmente respecto de las $(2 \mathrm{n}+1)$ correcciones.

$$
\begin{aligned}
& \frac{\partial I \cdot(x, \lambda)}{\partial \Delta L_{A}}=0=2 \Delta L_{A}+\lambda \Rightarrow \Delta L_{A}=-\frac{\lambda}{2} \ldots \ldots \ldots \ldots \ldots \ldots \ldots \ldots \ldots \ldots \ldots \ldots \ldots \ldots \ldots \ldots \ldots \\
& \frac{\partial L(x, \lambda)}{\partial \Delta L_{1}}=0=2 \Delta L_{1}-\lambda \emptyset_{1}+\lambda_{1}, \emptyset_{1} \Rightarrow \Delta L_{1}=\frac{1}{2}\left(\lambda-\lambda_{1}\right), \emptyset_{1} \ldots \ldots \ldots \ldots \ldots
\end{aligned}
$$

Si reemplazamos las ecuaciones del paso (2.5.2) en (2.5.1) y ordenamos debidamente, obtenemos:

$$
\begin{aligned}
& -2 \Delta M-\left(1+\emptyset_{1}^{2}+\emptyset_{2}^{2}\right) \lambda-\emptyset_{1}^{2} \cdot \lambda_{1}-\emptyset_{2}^{2} \cdot \lambda_{2} \\
& -2 \Delta M_{1}=-\emptyset_{1}^{2} \cdot \lambda+\left(\emptyset_{1}^{2}+\emptyset_{3}^{2}+\emptyset_{4}^{2}\right) \lambda_{1}-\emptyset_{2}^{2} \cdot \lambda_{2}-\emptyset_{4}^{2} \cdot \lambda_{4} \\
& -2 \Delta M_{2}=-\varrho_{2}^{2} \cdot \lambda+\left(0_{2}^{2}+\emptyset_{5}^{2}+\emptyset_{6}^{2}\right) \lambda_{2}-\emptyset_{5}^{2} \cdot \lambda_{5}-\emptyset_{6}^{2} \cdot \lambda_{6}
\end{aligned}
$$

Estas últimas ecuaciones se permite expresar mediante una ecuación lineal matricialmente: A ${ }_{\mathrm{n}, \mathrm{n}} \cdot \mathrm{X}_{\mathrm{n}, 1}=\mathrm{B}_{\mathrm{n}, 1}$; de quien se obtendrá los " $\mathrm{n}$ " multiplicadores de Lagrange.
Cálculo de los “n” Multiplicadores de Lagrange

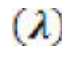

Expresión matricial mediante la ecuación lineal:

$$
A_{n, n} \cdot X_{n, 1}=B_{n, 1} \text { y será mostrado a continuación: }
$$



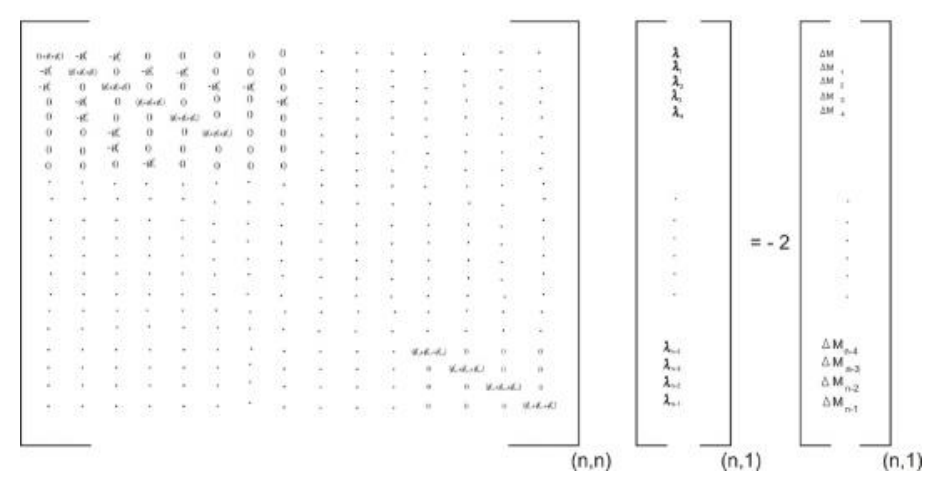

A continuación se procede a despejar la ecuación matricial:

$\mathrm{X}_{\mathrm{n}, 1}=\mathrm{A}_{\mathrm{m}, \mathrm{n}}^{\prime} \cdot \mathrm{B}_{\mathrm{n}-1 \text {; }}$ de donde se obtendrá los " $\mathrm{n}$ " Multiplicadores de Lagrange.

$$
\begin{aligned}
& \mathrm{L}_{1 \mathrm{c}}=\mathrm{L}_{1}-\mathrm{L}_{1} \\
& \mathrm{~L}_{2 \mathrm{c}}=\mathrm{L}_{2}-\mathrm{L}_{2}
\end{aligned}
$$

\section{OBTENCION DE LAS CORRECCIONES}

Después de haber calculado los Multiplicadores de Lagrange, se hallaran los " $(2 n+1)$ " correcciones; mediante las ecuaciones procedentes del paso (2.5.2)

Finalmente se corrigen las leyes:

Datos corregidos $=$ Datos - Correcciones

$\mathrm{L}_{\mathrm{Ac}}=\mathrm{L}_{\mathrm{A}}-\mathrm{L}_{\mathrm{A}}$

\section{APLICACIÓN INDUSTRIAL}

En la figura $\mathrm{N}^{\circ} 2$ se muestra el circuito de flotación bulk y sus respectivas leyes en la Tabla 2 .

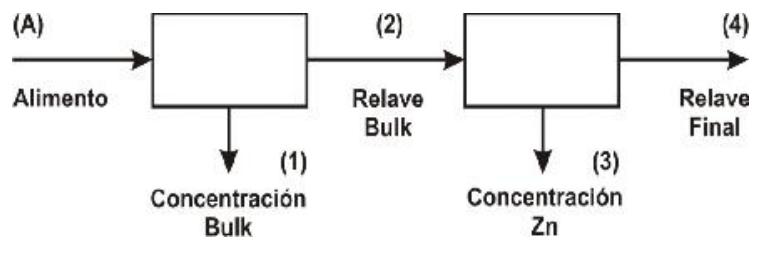

Fig. 2 Circuito de flotación Bulk.

\begin{tabular}{|c|c|c|c|c|c|c|c|}
\hline & & & & $L_{u}^{(i)}$ & & & \\
\hline Flujo (u) & $\begin{array}{c}\mathrm{Cu} \\
(\%)\end{array}$ & $\operatorname{Zn}(\%)$ & $\operatorname{Ag}(g / t)$ & $\mathbf{B i}(\mathrm{g} / \mathrm{t})$ & $\begin{array}{l}\text { Mo } \\
(\%)\end{array}$ & $\mathrm{Fe}(\%)$ & $\begin{array}{c}\mathbf{P b} \\
(\%)\end{array}$ \\
\hline A. Alimento & 1.88 & 3.504 & 61.86 & 237.808 & 0.012 & 5.456 & 0.023 \\
\hline 1. Conc.Bulk & 42.28 & 6.714 & 1236.089 & 5319.722 & 0.06 & 15.811 & 0.432 \\
\hline 2. Relave.Bulk & 0.175 & 0.241 & 11.219 & 24.048 & 0.007 & 5.196 & 0.006 \\
\hline 3. Conc.Zinc & 2.018 & 58.285 & 84.88 & 242.686 & 0.054 & 2.397 & 0.034 \\
\hline 4. Relave.Gen. & 0.1 & 0.138 & 9.9 & 20.76 & 0.002 & 9.87 & 0.002 \\
\hline
\end{tabular}

Tabla 2. Leyes de los diversos flujos. 
Balance Metalúrgico antes de ser corregidas las leyes

Tabla 3. De los flujos y leyes, estos últimos no concuerdan debido a la ausencia de sus correcciones.

\begin{tabular}{lrrrrrrrr}
\hline & $\mathbf{C u}(\boldsymbol{\%})$ & $\mathbf{Z n}(\boldsymbol{\%})$ & $\mathbf{A g} \%()$ & $\mathbf{B i}(\boldsymbol{\%})$ & $\begin{array}{l}\text { Mo } \\
(\boldsymbol{\%})\end{array}$ & $\mathbf{F e}(\boldsymbol{\%})$ & $\mathbf{P b}(\%)$ \\
\hline A & 100,000 & 100,000 & 100,000 & 100,000 & 100,000 & 100,000 & 100,000 \\
F1 & 90,965 & 7,750 & 80,824 & 90,482 & 20,224 & 11,722 & 75,972 \\
F2 & 8,932 & 6,600 & 17,403 & 9,703 & 55,974 & 91,383 & 25,032 \\
F3 & 0,525 & 8,130 & 0,671 & 0,499 & 2,199 & 0,215 & 0,722 \\
F4 & 5,078 & 3,760 & 15,278 & 8,334 & 15,911 & 172,700 & 8,301 \\
\hline
\end{tabular}

Balance Metalúrgico después de ser corregidas las leyes

Tabla 3. Los flujos y sus ya concuerdan debido a las correcciones cometidas.

\begin{tabular}{rrrrrrrr}
\hline & $\mathbf{C u}(\%)$ & Zn (\%) & Ag \%() & Bi (\%) & Mo (\%) & Fe (\%) & Pb (\%) \\
\hline A & 100,00 & 100,00 & 100,00 & 100,00 & 100,00 & 100,00 & 100,00 \\
F1 & 92,09 & 14,82 & 81,80 & 90,64 & 28,69 & 8,99 & 79,83 \\
F2 & 7,91 & 85,18 & 18,20 & 9,36 & 71,31 & 91,01 & 20,17 \\
F3 & 0,53 & 15,39 & 0,68 & 0,50 & 3,11 & 0,16 & 0,76 \\
F4 & 7,38 & 69,79 & 17,52 & 8,86 & 68,20 & $\mathbf{9 0 , 8 4}$ & 19,41 \\
\hline
\end{tabular}

\section{RESULTADOS Y DISCUSIONES}

Bondades que presenta el modelamiento matemático.

- Acoge y regula (gobierna) a todo el circuito (etapas de flotación de minerales), conjuntamente con sus leyes correspondientes.

- De calcular los flujos normalizados, posteriormente los multiplicadores de Lagrange y finalmente las correcciones respectivamente. $\mathrm{Y}$ con las leyes ya corregidas recién procedemos al balance metalúrgico,

- Se observa la coherencia existente en cada contenido metálico, a través de los flujos en el circuito (tabla 4).

- Caso contario se da en la tabla 3, donde el contenido metálico en la alimentación A no está de acorde con el contenido del mismo en los flujos F1 y F2; tampoco el flujo F2 lo hace con los flujos F3 y F4 en cuanto a sus leyes respectivamente. Así mismo es notorio 
identificar el valor de $172.70 \%$ del Fe en el relave (mayor al $100 \%$ )

\section{CONCLUSIONES}

En todo proceso continuo (nivel industrial), los parámetros operativos fluctúan dentro de un rango de estabilidad, motivo por el cual se hace indispensable realizar las correcciones del caso. Además los datos ya corregidos no se dan exactamente lo que realmente ocurre en el proceso, pero si tendrá la consistencia matemática y aproximándose a los valores reales.

Con lo cual queda demostrado que es posible operar en todo proceso de flotación de minerales a nivel industrial. Y que luego de identificar su circuito (diagrama de flujo), y sus leyes se le puede habituar al modelo (función generalizada), restringiéndole de acuerdo a parámetros operativos del proceso en cuestión, en donde cada celda con su respectivo flujo alimento, concentrado y relave e involucrado a su correspondiente ley, sea corregida.

Las correcciones por multiplicadores de Lagrange, ameritan ser lo más óptimo posible; de tal modo que las leyes ya corregidas cumplan con el contenido metálico: de lo que ingresa debe ser igual a lo que sale.
El modelamiento en mención es ya aplicable a los análisis granulométricos y con mucha afinidad también al complejo campo del flujo vehicular.

\section{AGRADECIMIENTO}

A mis colegas de la Facultad de Ingeniería Metalúrgica de la Universidad Nacional de Ingeniería, que aún me acogen.

\section{REFERENCIAS}

1. Lynch, A. J., "Circuito de trituración y molienda Edit. Rocas y minerales. 1ra. Edición 1980.

2. Ayres Hidalgo, F. A., Torres Ponce, M. T., "Técnicas matemáticas aplicadas al balance de materia".

3. Kobashikawa Chinen, J. A., "Corrección de análisis granulométricos y químicos por multiplicadores de Lagrange". Octubre 2003.

4. Gutiérrez R., L., Sepúlveda, J. E., "Dimensionamiento y optimización de plantas concentradoras mediante técnicas de modelación matemática". Centro de investigación minera metalúrgica, 1986.

5. Wils, B., Naiper, T. J., Munn, Octubre 2006.

Correspondencia: ademaredgar@yahoo.con, 